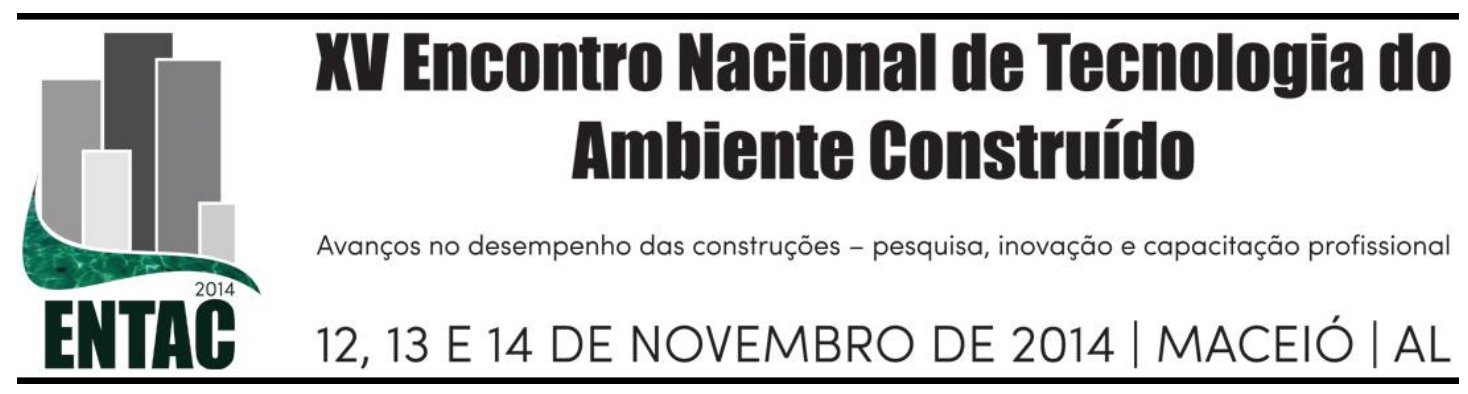

\title{
ACEITABILIDADE E CONFORTO TÉRMICO: CASO ESCOLA POLITÉCNICA DA UFBA
}

\author{
MORAES, Júlia S. de (1); BITTENCOURT, Leonardo S. (2); BATISTA, \\ JulianaOliveira (3)
}

(1) Doutorado Cidades - Universidade Federal de Alagoas - UFAL, e-mail: julia_arq@yahoo.com.br (2) Faculdade de Arquitetura - UFAL, e-mail: 1sb54@ hotmail.com, (3) Faculdade de Arquitetura - UFAL, email: juliana.batista@ fau.ufal.br

\begin{abstract}
RESUMO
Estudos recentes sobre conforto térmico em climas quentes e úmidos sugerem uma aceitabilidade da velocidade do ar na ordem de até $1 \mathrm{~m} / \mathrm{s}$ no interior de ambientes, não ocorrendo queixas quando a velocidade do ar excedeu esse valor (CÂNDIDO et. al, 2008). Essa velocidade é superior ao valor sugerido pela ASHRAE 55 (2004), onde a velocidade de $0,2 \mathrm{~m} / \mathrm{s}$ foi considerada como o limite máximo. Este trabalho tem o objetivo de verificar a aceitabilidade quanto ao conforto térmico em edifícios universitários. O método adotado consiste na análise da relação entre a aceitabilidade do movimento do ar e conforto térmico no interior dos edifícios ventilados naturalmente, utilizando como base estudos de pósocupação do prédio da Escola Politécnica da UFBA. Os resultados de diversos índices de conforto foram comparados com a opinião dos usuários. Os resultados encontrados confirmam os estudos de CÂNDIDO e outros (2008) quanto à aceitabilidade do aumento da velocidade do ar em ambientes internos. No caso do edifício estudado, o indicador PMV apresenta uma situação de levemente quente, tanto no verão como no inverno e o PPD apresenta, em média, 28\% de pessoas insatisfeitas durante o ano. As variáveis climáticas plotadas no Nomograma de Temperatura Efetiva indicaram zona de conforto para a situação estudada. Quanto à opinião dos usuários, a maioria achou a temperatura da sala confortável, porém, consideraram que a ventilação da sala de aula era baixa, demandando um incremento da velocidade do ar proporcionada pelos ventiladores. $\mathrm{O}$ estudo sugere uma afinidade entre os indicadores quantitativos e qualitativos e ratifica a estratégia de aumento da movimentação de ar no interior dos ambientes para obtenção de conforto em edificações universitárias localizadas em climas quente e úmido.
\end{abstract}

Palavras-chave: Aceitabilidade do movimento do ar, Ventilação natural, Conforto Térmico.

\begin{abstract}
Recent studies on thermal comfort in hot and humid climates suggest acceptability of air velocity on the order of $1 \mathrm{~m} / \mathrm{s}$ within environments, no complaints occurring when the air velocity exceeded this value (CÂNDIDO et al. 2008). This speed is higher than the value suggested by the ASHRAE 55 (2004), where the speed of $0.2 \mathrm{~m} / \mathrm{s}$ was considered as the upper limit. This study aims to verify the acceptability regarding thermal comfort in university buildings. The method adopted consists in analyzing the relationship between the acceptability of air movement and thermal comfort inside the naturally ventilated buildings, based on studies using post-occupancy methods applied to the building of the Polytechnic School UFBA. The results of various comfort indices were compared with the opinion of the users. Results confirm the studies of CÂNDIDO and others (2008) about the acceptability of increased air velocity indoors. In the case of the studied building, the PMV indicator presents a situation slightly hot, both in summer and in winter and PPD has on average, $28 \%$ of dissatisfied people during the year. Climate variables plotted on the Effective Temperature Nomogram indicated comfort zone for the situation studied. As for the opinion of users, the majority found the temperature of the room comfortable, however, found that ventilation of the classroom was low, requiring an increase in the air velocity provided by fans. The study suggests an affinity between the quantitative and qualitative indicators and
\end{abstract}


confirms the strategy of increasing air movement inside the environment to obtain comfort in university buildings located in hot and humid climates.

Keywords: Acceptability of air movement, Natural Ventilation, Thermal Comfort.

\section{INTRODUÇÃO}

O aproveitamento da ventilação natural nas edificações e implicações no espaço habitado vem sendo estudado como uma importante estratégia para obtenção do conforto térmico.

Dos primeiros trabalhos desenvolvidos por Beaufort, no século XIX, que classificam a intensidade dos ventos na célebre Escala de Beaufort, até a carta bioclimática de Olgyay (1963), que adapta os princípios de Beaufort para o ambiente habitado, o vento e sua intensidade no interior dos ambientes foram, ao longo dos tempos, classificados em índices, escalas e tabelas que expressam a sensação de conforto (GIVONI, 1976), (FANGER, 1972).

Os índices de conforto térmico, em sua maioria, "expressam a sensação de conforto como uma temperatura efetiva ou temperatura operativa que combina o efeito da temperatura do ar, umidade, radiação e movimento do ar". (BITENCOURT; CÂNDIDO, 2008. p. 13).

Como exemplo, o Nomograma de Temperatura Efetiva, proposto por Yaglow e Houghten (1923), demonstra a correlação entre as sensações de conforto e as condições de temperatura, umidade e velocidade do ar, apresentando as condições de conforto térmico. O índice foi posteriormente corrigido por Vernon e Warner (1932), utilizando a temperatura do termômetro de globo em vez de temperatura seca do ar. (FROTA; SCHIFFER, 2001)

Outros índices foram desenvolvidos a partir de análise estatística das condições térmicas preferidas como o Voto Médio Predito - PMV e Porcentagem de Pessoas Insatisfeitas PPD (INTERNATIONAL, ... 2005).

Atualmente existem estudos tentando definir a aceitabilidade da velocidade do ar, quando e onde o movimento do ar é desejável, a partir da obtenção da sensação do usuário e conforto térmico (CÂNDIDO et al., 2008) (SANTAMOURIS, 2004) (TOFTUM, 2004), (FANGER, 1988).

Segundo Bittencourt e Cândido (2008), a velocidade máxima do ar considerada como aceitável pode variar entre 0,5 e $2,5 \mathrm{~m} / \mathrm{s}$. O limite máximo é baseado em problemas práticos ocasionados com o movimento do ar, tais como vôo de papel sobre a mesa ou desarranjo de penteados.

Para os autores, o incômodo de um fluxo de ar constante pode ser verdadeiro em climas frios, mas não reflete a realidade de climas quentes e úmidos, onde a ventilação é um fator fundamental na determinação do conforto térmico. (BITENCOURT e CẨNDIDO, 2008).

A literatura sobre o conforto térmico indica que a velocidade do ar aceitável no interior em climas quentes deve variar 0,2 e 1,50 m/s. A ASHRAE 55 (2004) estabelece o valor de $0,2 \mathrm{~m} / \mathrm{s}$ como limite máximo da velocidade do ar no interior de edifícios com ar condicionado, onde os ocupantes não têm controle direto sobre o ambiente. No entanto, estudos recentes com usuários de edificações universitárias no clima quente e úmido de Maceió (AL), apresentam resultados onde sob temperaturas operativas acima de $24{ }^{\circ} \mathrm{C}$, a velocidade média do ar preferida é de até $1 \mathrm{~m} / \mathrm{s}$, não ocorrendo queixas quanto a velocidades do ar excedeu este limite (CÂNDIDO et al., 2008). 
Em casos onde se pretendem discutir limites e sua a aceitabilidade, a avaliação pósocupação torna-se um importante instrumento. Estudos técnicos relacionados à opinião do usuário geram conhecimento sobre a relação ambiente-comportamento, permitindo elaborar recomendações para o próprio estudo de caso, ou para futuros projetos semelhantes (ORNSTEIN et al., 1995).

Este trabalho tem o objetivo de investigar se aceitabilidade quanto ao conforto térmico em edifícios universitários se repete em outras localidades do nordeste brasileiro, utilizando como referencia a base de dados climáticos do Caso Politécnica (MORAES, 2007), desenvolvida em um período próximo ao estado de Maceió.

Pretende-se também examinar a aceitabilidade quanto aos índices de conforto PMV, PPD e Nomograma de Temperatura Efetiva, comparando-se os resultados destas predições com as opiniões declaradas pelos usuários.

\section{PROCEDIMENTOS METODOLÓGICOS}

Para alcançar os objetivos acima delineados, foi realizada uma análise da relação entre o movimento do ar e conforto térmico no interior dos edifícios ventilados naturalmente. Foram computados os valores medidos em uma pesquisa realizada no prédio da Escola Politécnica da Universidade Federal da Bahia - UFBA descrita em Moraes (2007), tendo sido desenvolvidos os seguintes procedimentos:

(I) Caracterização climática da cidade de Salvador e do microclima no interior da edificação; (II) Descrição das variáveis ambientais medidas na edificação; (III) Avaliação qualitativa dos usuários da edificação; (IV) Aplicação dos índices de conforto: PMV, PPD e Nomograma de Temperatura Efetiva.

\subsection{Caracterização climática da cidade de Salvador e do microclima no interior do edifício}

A cidade de Salvador está localizada na latitude de $12^{\circ} 52^{\prime}$ Sul. Encontra-se na costa nordeste brasileira, banhada pelo Oceano Atlântico.

Possui o clima quente e úmido, tipicamente tropical, com temperatura do ar média anual de $25,2{ }^{\circ} \mathrm{C}$ e umidade relativa média anual de 80,8\% (FREIRE et al., 1997). Os ventos predominantes são: os alísios de sudeste, na maior parte do ano, os ventos leste, na primavera e verão e ventos nordeste, de outubro a março (VALENTE, 1977).

Salvador é uma cidade bastante úmida, com baixa amplitude térmica. Segundo Freire e outros (1997, p.127), a "condição climática da Cidade de Salvador promove a sensação térmica de estresse térmico positivo durante todo o ano, amenizada nos meses junho, julho e agosto".

A Escola Politécnica está localizada em uma cumeada da Cidade de Salvador, no bairro da Federação, campus Ondina - Federação e está implantada a 72 metros de altitude em relação ao nível do mar (Figura 01).

Figura 1- Salvador (Bahia) destacando-se a localização da Escola Politécnica 


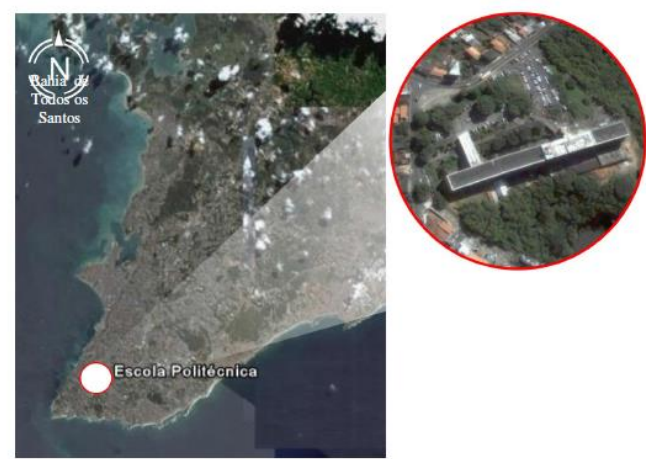

Fonte: Google Earth, adaptado por Moraes (2007).

A situação privilegiada, sem obstruções do entorno, associada à forma alongada com implantada com orientação noroeste - sudeste propicia ventilação e iluminação natural sem obstruções.

A edificação foi concebida de forma a receber a ventilação cruzada e é dotada de elementos que reduzem a incidência de radiação solar no interior da edificação na maior parte dos recintos. A fachada principal (noroeste) é constituída por cobogós nos trechos de circulação e a fachada posterior (sudeste) apresenta esquadrias em fita protegida por marquise e venezianas (Figura 02).

Pode-se dizer que o edifício da Escola Politécnica está em consonância com as recomendações bioclimáticas para Salvador (GIVONI, 1992).

Figura 2-Escola Politécnica da UFBA. Cobogós da fachada noroeste e marquise com venezianas na fachada sudeste

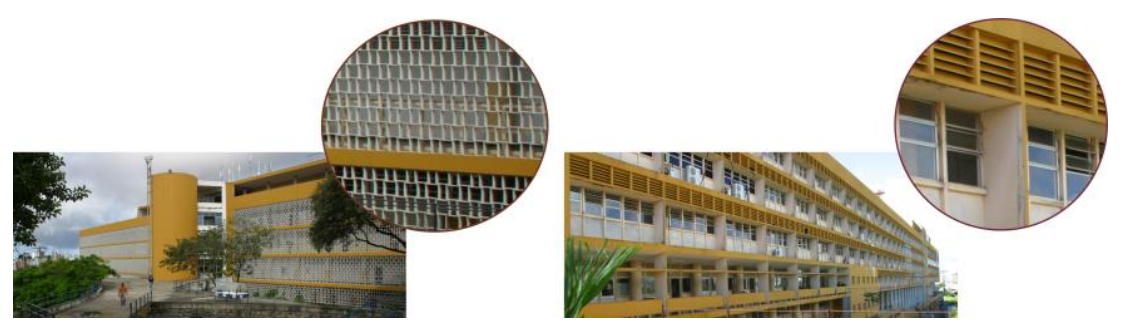

Foto: Antônio Saturnino, adaptada por Moraes (2007).

\subsection{Descrição das variáveis medidas nos ambientes}

No banco de dados realizado por Moraes (2007) constam duas séries de medições. Para realização das mesmas, foram utilizadas duas estações meteorológicas móveis da marca SMETEK- System, cedida pelo Laboratório de Conforto Ambiental da Faculdade de Arquitetura da UFBA.

As estações foram posicionadas uma no interior e outra no exterior da edificação. As estações mediram: a temperatura do ar - Ta $\left({ }^{\circ} \mathrm{C}\right)$, a umidade relativa do ar - UR (\%), a velocidade do ar $-\mathrm{V}(\mathrm{m} / \mathrm{s})$, a temperatura de globo $-\mathrm{TG}\left({ }^{\circ} \mathrm{C}\right)$ e a radiação global $\left(\mathrm{W} / \mathrm{m}^{2}\right)$, em conformidade com a - ISO 7726 (1996).

As medições ocorreram diariamente, no período de 8 às 20 horas, com registros efetuados em intervalos de trinta minutos. Nos ambientes internos, foram realizadas medições da velocidade do ar (em m/s) com anemômetro às 8, 12, 16 e 18 horas. Ambos os instrumentos possuíam certificados de calibração. 
As medições ocorreram nos dias 21, 22 e 23 de março de 2005 e nos dias 31 de agosto, 01 e 02 setembro de 2005. As datas foram definidas em função do calendário escolar, de acordo com a disponibilidade de obtenção dos aparelhos de medição.

A sala de aula definida para esse estudo é a de número 07.01.05. Encontra-se no sétimo andar, bloco 1. É uma sala representativa do projeto original da edificação, pois não sofreu reformas, possui esquadrias em fita voltadas para o sudeste e cobogós na circulação (Figura 03). As medições ocorreram nos dias 23 de março e 31 de agosto de 2005.

Figura 3-Localização da sala 07.01.05.

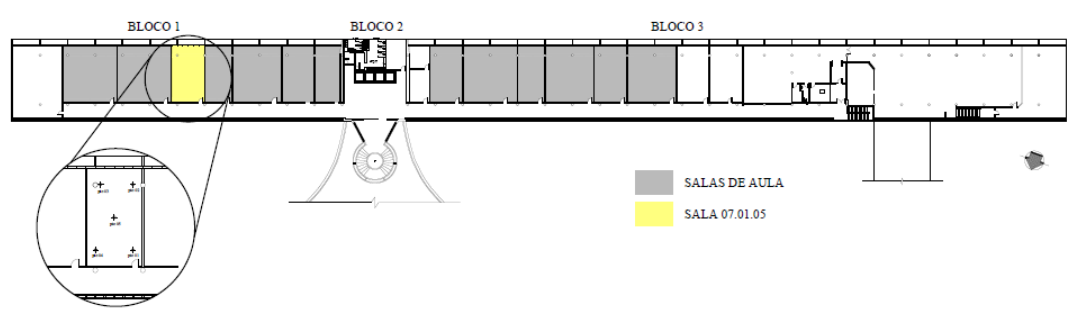

Fonte: Autor.

\subsection{Opinião do usuário}

Com o objetivo de obter a opinião coletiva quanto ao desempenho energético e térmico da edificação foi realizada uma consulta com os usuários da edificação.

A enquete foi desenvolvida por Roméro (1997) e aplicada de forma adaptada em instituições de ensino na cidade de Salvador (CARVALHO; MORAES, 2002). Possui 11 perguntas de natureza subjetiva e objetiva. As questões abordam temas como qualidade da iluminação, sensação de calor, uso da energia elétrica, comportamental e outros aspectos.

No banco de dados disponibilizado por Moraes (2007) constam 369 enquetes realizadas nos oito andares da Escola, seguindo procedimentos estatísticos descritos no trabalho. Para este estudo, foram utilizadas 4 perguntas do questionário aplicado por Moraes (2007), que se referem especificamente ao conforto térmico (Quadro 1).

\section{Quadro1-Enquete}

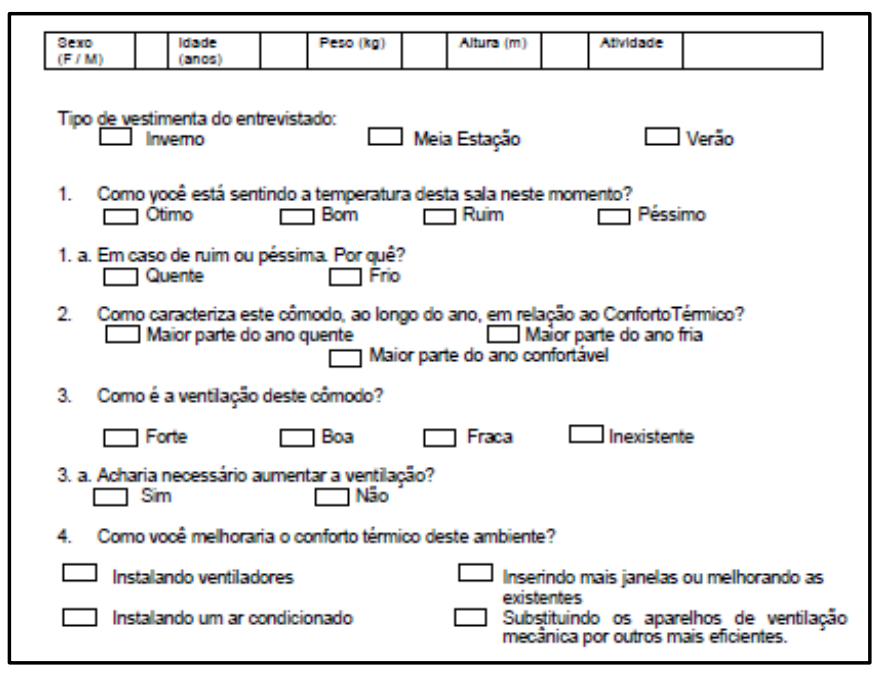


Fonte: Romero (1997).

As consultas aos usuários foram feitas durante as medições, com os alunos que se encontravam utilizando o recinto em atividade de aula. Nos dias e horários de medição, apresentados no item 2.2, foram realizados um total de 148 enquetes.

O usuário médio da sala de aula é jovem, alto e sem sobrepeso. Têm em média 24 anos, 70,4 quilos e 1,73 metros de altura e predominância do sexo masculino.

\subsection{Variáveis subjetivas}

As condições climáticas, local de trabalho, as vestimentas e atividade física exercida são aspectos que interferem diretamente na opinião do usuário.

A roupa é uma variável que influencia na opinião do usuário, pois é responsável pela sensação de conforto térmico. "A pele troca calor por condução, convecção e radiação com a roupa, que por sua vez troca calor com o ar por convecção e com outras superfícies por radiação.” (LAMBERTS et al., 1997).

A ISO 7730 (2005) quantifica a resistência térmica das vestimentas, atribuindo uma escala de valores medida em clo, clothing em inglês. Associado ao clo, a atividade exercida no ambiente também afeta a sensação de conforto térmico. A ISO 7730 (2005) também apresenta valores de taxa metabólica correspondentes a diferentes atividades físicas, quantificadas em Watts $/ \mathrm{m}^{2}$.

O usuário tipo foi considerado do sexo masculino, com isolamento de vestimenta correspondente a 0,645 clo (com camisa curta, calça média, sapatos com meia fina e cueca). A atividade exercida é a de escritório, com $70 \mathrm{~W} / \mathrm{m}^{2}$ de taxa metabólica.

Os procedimentos para obtenção do Índice de Temperatura Efetiva estão descritos em Frota e Schiffer (2001). Foi considerada a zona de conforto térmico para pessoas normalmente vestidas, em trabalho leve e considerando o usuário como habitante de regiões de climas quentes.

\subsection{Voto Médio Predito - PMV e Porcentagem de Pessoas Insatisfeitas - PPD}

A sensação térmica percebida pelo indivíduo é representada pelo "PMV" ou voto médio predito. A sensação térmica é representada na escala sétima de sensações, que oscila entre "muito frio" (-3) e "muito calor" (+3). O valor " 0 " representa neutralidade térmica, ou seja, ausência de desconforto. Já o índice PPD estabelece a quantidade estimada de pessoas insatisfeitas termicamente com o ambiente, sendo $10 \%$ o valor considerado aceitável (ISO 7730, 2005).

Para obtenção dos Índices PMV e PPD foi utilizado o Software ANALISES 1.5 (LABEEE, 2013). Este software permite a avaliação de dados climáticos segundo o modelo de Fanger (1972). Os dados de entrada consideram as características subjetivas (peso, sexo e idade), a vestimenta, a atividade exercida e as variáveis ambientais do local em estudo. Como dados de saída obtêm-se os indicadores PMV e PPD, em conformidade com a ASHRAE 55 (2004).

\section{RESULTADO E DISCUSSÃO}

No caso da sala examinada, as variáveis ambientais obtidas encontram-se situadas na zona de conforto do Nomograma de Temperatura Efetiva (Figura 04), sendo os pontos plotados na cor vermelho representam o dia quente, medido no mês de março e os azuis, o dia frio, medido no mês de agosto. 
As temperaturas obtidas na sala variam entre $27,7{ }^{\circ} \mathrm{C}$ e $29,4^{\circ} \mathrm{C}$, com umidade relativa média de $75 \%$, medidos em março. Em agosto, a temperatura variou entre $25,5^{\circ} \mathrm{C}$ e $26,7^{\circ} \mathrm{C}$, com umidade relativa média de $72 \%$ (Tabela 1 ).

Figura 4 -Nomograma de Temperatura Efetiva

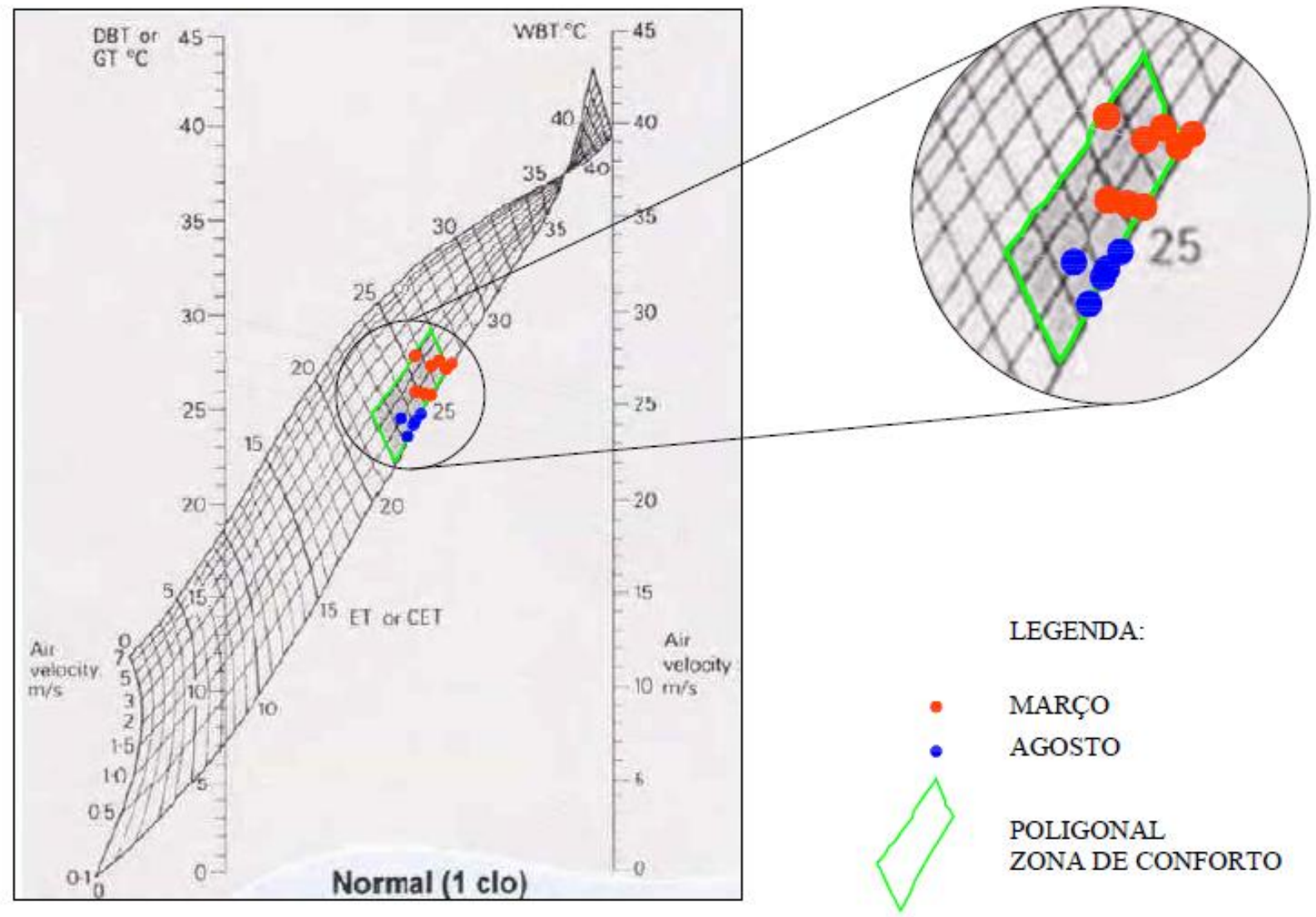

Fonte: Autor.

Tabela 1 -Resumo das variáveis ambientais medidas na sala 07.01.05

\begin{tabular}{|c|c|c|c|c|c|c|c|c|c|c|}
\hline \multicolumn{2}{|c|}{ Período } & \multirow{3}{*}{$\begin{array}{c}\text { TEMP1 } \\
{ }^{\circ} \mathrm{C}\end{array}$} & \multirow{3}{*}{$\frac{\mathbf{U R}}{\%}$} & \multirow{3}{*}{$\frac{\text { Tef }}{{ }^{\circ} \mathrm{C}}$} & \multicolumn{5}{|c|}{ VEL AR } & $\begin{array}{c}\text { Sala } \\
07.05 .01 \\
\end{array}$ \\
\hline \multirow{2}{*}{$\stackrel{\pi}{\ddot{a}}$} & \multirow{2}{*}{ HORA } & & & & $\mathrm{m} / \mathrm{s}$ & $\mathrm{m} / \mathrm{s}$ & $\mathrm{m} / \mathrm{s}$ & $\mathrm{m} / \mathrm{s}$ & $\mathrm{m} / \mathrm{s}$ & \multirow{3}{*}{$\underset{\text { pto } 03}{4} \underset{\text { pto } 02}{4}$} \\
\hline & & & & & Pto. 1 & Pto. 2 & Pto. 3 & Pto. 4 & Pto. 5 & \\
\hline \multirow{4}{*}{$\begin{array}{l}0 \\
\frac{0}{0} \\
8 \\
\frac{00}{4}\end{array}$} & 09:00 & 25,5 & 77 & 23,4 & 0 & 0 & 0 & 0,1 & 0 & \\
\hline & 12:00 & 26,3 & 77 & 24,4 & 0 & 0 & 0,36 & 0 & 0 & \multirow{2}{*}{$\underset{\text { pto } 05}{+}$} \\
\hline & 15:00 & 26,7 & 64 & 23,9 & 0 & 0 & 0 & 0,31 & 0 & \\
\hline & 18:00 & 26,2 & 71 & 24,1 & 0,6 & 0 & 0 & 0,3 & 0,25 & \multirow[b]{2}{*}{ 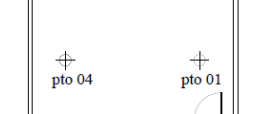 } \\
\hline \multirow{4}{*}{ 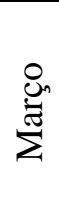 } & 09:00 & 27,7 & 76 & 25,4 & 0,4 & 0,2 & 0,3 & 0 & 0,3 & \\
\hline & 12:00 & 29,4 & 72 & 26,5 & 0 & 0 & 0 & 0 & 0 & \multirow{3}{*}{ CiRCULAÇÃo } \\
\hline & 15:00 & 29,2 & 74 & 26,6 & 1,5 & 0,2 & 0 & 0,1 & 0,3 & \\
\hline & $18: 00$ & 28,7 & 77 & 26,2 & 0,6 & 0,8 & 0,2 & 0,2 & 0,1 & \\
\hline
\end{tabular}

Sendo:

TEMP 1 = Temperatura; UR = Umidade Relativa; Tef $=$ Temperatura Efetiva; VEL AR = Velocidade do ar no ponto. 
Tabela 2 - Resultados da consulta ao usuário e aplicação do índice PMV e PPD

\begin{tabular}{|c|c|c|c|c|c|c|c|c|c|c|c|c|c|c|c|c|c|}
\hline \multicolumn{2}{|c|}{ Período } & \multicolumn{14}{|c|}{$\begin{array}{c}\text { Resultados da consulta ao usuário } \\
\text { (Perguntas e Percentuais de respostas) }\end{array}$} & \multicolumn{2}{|c|}{$\begin{array}{l}\text { Índice } \\
\text { Fanger }\end{array}$} \\
\hline \multirow[b]{2}{*}{ 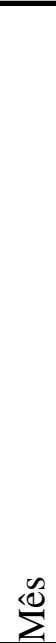 } & \multirow[b]{2}{*}{$\underset{0}{\tilde{0}}$} & \multicolumn{4}{|c|}{ P 1} & \multicolumn{2}{|c|}{ P 1.a } & \multicolumn{3}{|c|}{ P 3} & \multicolumn{5}{|c|}{ P 4} & \multirow[b]{2}{*}{$\begin{array}{l}\stackrel{\rho}{e} \\
\sum_{a}^{2}\end{array}$} & \multirow[b]{2}{*}{$\frac{\hat{d}}{\hat{a}}$} \\
\hline & & $\begin{array}{l}\widehat{o} \\
o \\
. \vdots \\
.0\end{array}$ & 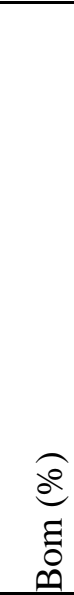 & 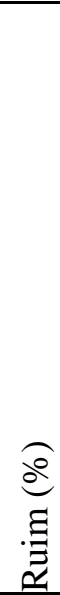 & 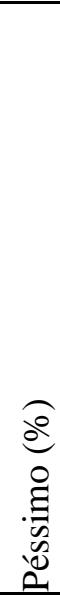 & 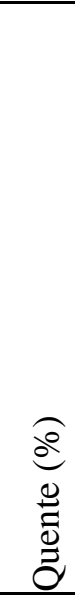 & 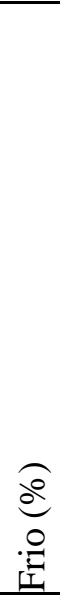 & $\begin{array}{l}\widehat{d} \\
0 \\
0 \\
0 \\
0 \\
0\end{array}$ & $\begin{array}{l}\text { of } \\
\stackrel{0}{0}\end{array}$ & 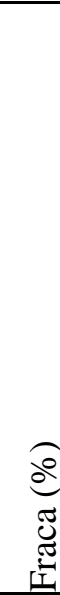 & 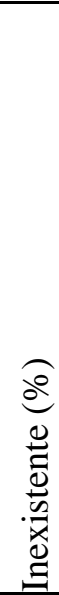 & 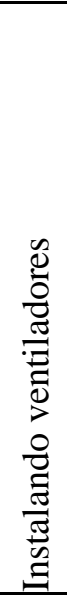 & 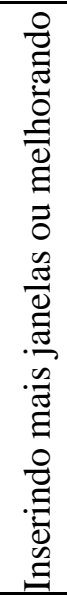 & 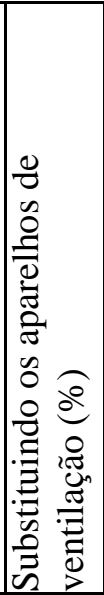 & 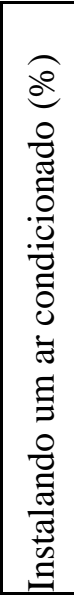 & & \\
\hline \multirow{4}{*}{$\begin{array}{l}0 \\
\stackrel{0}{0} \\
80 \\
\&\end{array}$} & 09:00 & \begin{tabular}{|l|}
9,1 \\
\end{tabular} & 64 & 27 & 0 & 67 & 33 & 27 & 18 & 36 & 9,1 & 9,1 & 27 & 55 & 9,1 & 1,1 & 34 \\
\hline & 12:00 & \begin{tabular}{|l|}
29 \\
\end{tabular} & 64 & 7,1 & 0 & 67 & 33 & 0 & 31 & 54 & 0 & 7,1 & 7,1 & 79 & 7,1 & 1,2 & 38 \\
\hline & $15: 00$ & 5,6 & 67 & 28 & 0 & 75 & 25 & 11 & 17 & 44 & 11 & 11 & 44 & 33 & 11 & & \\
\hline & 18:00 & 33 & 67 & 0 & 0 & 0 & 0 & 22 & 26 & 30 & 13 & 0 & 56 & 39 & 5,6 & 0,6 & 13 \\
\hline \multirow{4}{*}{$\begin{array}{l}\text { o } \\
\text { : } \\
\sum^{\pi}\end{array}$} & 09:00 & 0 & 100 & 0 & 0 & 0 & 0 & 0 & 0 & 100 & 0 & 0 & 0 & 100 & 0 & 0,7 & 18 \\
\hline & 12:00 & 0 & 100 & 0 & 0 & 0 & 0 & 17 & 17 & 67 & 0 & 0 & 0 & 50 & 33 & 1,4 & 46 \\
\hline & 15:00 & 0 & 29 & 57 & 14 & 100 & 0 & 20 & 40 & 40 & 0 & 43 & 14 & 14 & 0 & 0,9 & 25 \\
\hline & 18:00 & 0 & 69 & 25 & 6,3 & 100 & 0 & 16 & 26 & 58 & 0 & 19 & 6,3 & 44 & 13 & 1 & 26 \\
\hline
\end{tabular}

Fonte: Autor.

Nota:

P 1:Como você está sentindo a temperatura desta sala neste momento?

P 1.a: Em caso de ruim ou péssima. Por quê?

P 3: Como é a ventilação deste cômodo?

P 4: Como você melhoraria o conforto térmico deste ambiente?

A opinião do usuário confirma as indicações de conforto apresentadas no Nomograma de temperatura efetiva. A maioria dos usuários acha a temperatura da sala no momento boa. Porém, informam que a ventilação da sala é fraca, indicando como soluções a melhoraria da eficiência das esquadrias e a substituição dos equipamentos mecânicos por outros com melhor desempenho (Tabela 2).

Comparando os dados da velocidade do ar com a opinião do usuário, na medição de março, às 15 horas e às 18 horas, registraram-se velocidades do ar superiores a 0,6 m/s no interior do recinto. Na condição onde a ventilação atingiu $1,5 \mathrm{~m} / \mathrm{s}$, às 15 horas, obteve-se o melhor percentual de aceitabilidade por parte dos usuários, onde $40 \%$ considerou a ventilação do recinto boa. (Tabela 2).

É importante ressaltar que a opção pelo uso do ar condicionado atingiu uma faixa entre $5,5 \%$ e $12 \%$ em todos os períodos, com exceção para o horário de 12 horas no mês de março, quando $33 \%$ dos usuários solicitaram o condicionamento artificial como alternativa para melhorar o conforto térmico no recinto (Tabela 2).

O índice PMV ratifica a opinião dos usuários, apresentando resultados variando entre 0,6 e 1,4, valores que configuram o ambiente em situação de "levemente quente", tanto no mês de março como em agosto (Tabela 2).

Já o PPD apresenta faixas entre $12,7 \%$ e 45,6 \% de pessoas insatisfeitas no recinto, a 
depender do horário e da estação do ano, sendo em média $28 \%$ de pessoas insatisfeitas quanto ao conforto térmico, valor próximo ao obtido com a opinião dos usuários, onde $7 \%$ a $31 \%$ usuários consideram a temperatura do ambiente ruim ou péssima, com exceção ao mês de março no horário de 15 horas, quando $71 \%$ dos usuários manifestaram-se insatisfeitos (Tabela 2).

\section{CONSIDERAÇÕES FINAIS}

No artigo, através do caso Escola Politécnica da UFBA, foi possível ratificar as indicações da literatura em edificações do tipo escolar no nordeste brasileiro que demostraram a aceitabilidade do aumento da velocidade do ar em ambientes internos, propiciando conforto térmico (CÂNDIDO et al., 2008) (SANTAMOURIS, 2004) (TOFTUM, 2004).

Os resultados demonstraram que as sugestões apresentadas pelos usuários para melhorar a sensação de conforto térmico no ambiente analisado estão relacionadas ao aumento da ventilação, pelo incremento de equipamentos de ventilação mecânica ou por melhorias nas esquadrias, com o objetivo de propiciar aumento da circulação do ar. As sugestões apresentadas contrariam a cultura vigente de instalação de sistemas de climatização artificial do tipo ar condicionado.

O estudo de caso também permitiu demonstrar a afinidade existente entre os indicadores quantitativos obtidos: plotagem das variáveis climáticas medidas no Nomograma de Temperatura Efetiva, que contém o indicativo de zona de conforto; a utilização do PMV e PPD e os indicadores qualitativos obtidos com a opinião dos usuários, principalmente no que se refere à aplicação em clima quente e úmido. A correspondência entre os indicadores confirmam a aplicação dos mesmos como ferramenta de avaliação pósocupação de edifícios escolares.

Espera-se que o estudo contribua para o estabelecimento de novos parâmetros de velocidade do movimento do ar no interior de edifícios, em prol do conforto térmico na região do nordeste brasileiro.

\section{REFERÊNCIAS}

AMERICAN SOCIETY OF HEATING, REFRIGERATING AND AIR CONDITIONING ENGINEERS - ASHRAE. Standard 55: Thermal environmental conditions for human occupancy. Atlanta, ASHRAE: 2004.

BITTENCOURT, Leonardo S. e CÂNDIDO, Christhina. Introdução à ventilação natural. Maceió: EDUFAL, 2008.

CÂNDIDO, Christhina, DEAR, Richard de, LAMBERTS, Roberto and BITTENCOURT, Leonardo. Natural ventilation and thermal comfort: air movement acceptability inside naturally ventilated buildings in Brazilian hot humid zone. Air Conditioning and the Low Carbon Cooling Challenge, Cumberland Lodge. Network for Comfort and Energy Use in Buildings London, p. 27-29, 2008.

CARVAlHO, Maria Lúcia ; MORAES, Júlia. Aplicação da Metodologia APO - Avaliação Pós - Ocupação, no Campus da UFBA, com vistas a implantação de um Programa de Gestão Ambiental $-2^{\mathbf{o}}$ etapa. Relatório Final de atividades apresentado ao Programa Institucional de Iniciação Científica do CNPq. Salvador, 2002.

FANGER, P. O. Thermal Comfort: analysis and applications in environment al engineering. New York: McGraw - Hill, 1972. 
FANGER, P. O., MELIKOV, A. K., HANZAWA, H. and RING, J. W. Air turbulence and sensation of draught. Energy and Buildings. Energy and Buildings, n. 121, p. 21-39, 1988.

FREIRE, Tereza ; NERY, J. M. F. G. ; CARVALHO, L. ; ANDRADE, Telma Côrtes Quadros de ; FREIRE, M. ; AZEVEDO, H. ; PIZARRO, E. . Primeira aproximação para estudo de clima urbano em Salvador. . In: Encontro Nacional de Conforto no Ambiente Construído, 1997, Salvador. Anais do IV Encontro de Conforto no Ambiente Construído ANCAC / ANTAC, 1997. v. 01. p. 124-133.

FROTA, Anésia Barros e SCHIFFER, Sueli R. Manual de conforto térmico: arquitetura, urbanismo. São Paulo: Studio Nobel, 2001.

GIVONI, B. Man, climate and architecture. London: Elsevier, 1976.

GIVONI, Baruch. Confort climate analysis and building design guidelines. Energy and Buildings, n. 18. [local?]: Elsevier, 1992.

INTERNATIONAL FOR ORGANIZATION STANDARDIZATION - ISO. ISO 7726 . Ergonomics of the thermal environment - Instruments for measuring physical quantities. Suíça: ISO, 1996.

ISO Standard 7730. Analytical determination and interpretation of thermal comfort using calculation of the PMV and PPD indices and local thermal comfort criteria. Geneva: ISO, 2005.

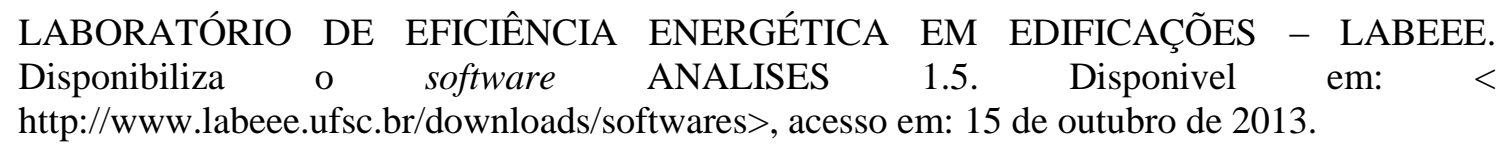

LAMBERTS, R; DUTRA, L; PEREIRA, F. Eficiência Energética na Arquitetura. São Paulo: PW Editores. 1997.

MORAES, J. S. Indicadores Energéticos de Edifícios Públicos: Estudo de Caso da Escola Politécnica - UFBA. Salvador: 2007. Dissertação (Mestrado em Gerenciamento e Tecnologias Ambientais no Processo Produtivo - ênfase em Produção Limpa) - Escola Politécnica, Universidade Federal da Bahia.

OLGYAY, V. Design with climate. New Jersey: Princeton University, 1963.

ORNSTEIN, Sheila Walbe; BRUNA, Gilda e ROMÉRO, Marcelo. Ambiente Construído \& Comportamento: A Avaliação Pós-Ocupação e a Qualidade Ambiental. São Paulo: Studio Nobel, 1995.

ROMERO, M. Avaliação Comportamental e Energética do Edifício da FAUUSP. In: Cadernos Técnicos Aut, n. 3. São Paulo: FAUUSP, 1997.

SANTAMOURIS, M. Adaptive thermal confort and ventilation. Ventilation Information Paper, Air Infiltration and Ventilation Centre. Belgium, Air Infiltration and Ventilation Centre, v. 12, 2004.

TOFTUM, J. Air movement - good or bad? Indoor Air. Vol. 14, Issue Supplement s7, p. 4045. [local?]: Indoor Air, 2004.

VALENTE, M. Conforto térmico em Salvador. Salvador: UFBA - Centro Editorial e Didático, 1977. 